\title{
VR 技术在建筑设计教与学中的应用初探
}

\author{
朱昊东 \\ 滨州学院建筑工程学院 \\ DOI:10.32629/er.v2i4.1787
}

[摘要] 随着中国社会经济的发展, VR 技术已经逐步走向了人们的生活,带给人们精神和经济上的富足,人们对于 VR 应用的 标准已经从以前的能适应,使用它获得生活的方便,走向 VR 技术对我们的衣食住行进行方方面面渗透的局面。在建筑教学层 面, 人们更加注重将现代化技术渗透到教育教学里面, 由此将 VR 技术应用到建筑设计的教与学中逐渐被人们所推崇。而且为 了让 VR 技术教学得到推广,让更多的人能够更好地学到这种应用技术,建筑工程层面的教育机制开始走向信息化,利用现代化 科技来完善知识体系的学习。

[关键词] VR 技术; 建筑教学; 应用; 初步探索; 虚拟技术

\section{前言}

随着时代的发展, 我们所面临的建筑项目规模逐渐巨大 化以及复杂化, 实现一个庞大而复杂的项目, 所需要的建筑 知识体系也必将是庞大的, 而且因为工程的分项过于繁多, 每一类的知识学习分类也是很难理清的, 如果按照以往传统 的学习方案, 学生的工作量不但庞大, 而且还很难接受。因此 做 VR 技术在建筑教学里面的应用, 通过虚拟模拟让学生能 够直面建筑的整体构造, 也就变成一个非常重要的事。本文 首先分析传统教育方式对于建筑设计学习产生的阻碍, 然后 初步探索了 VR 技术在建筑教学中的应用, 以及所做出的初 步设想。

\section{$1 \mathrm{VR}$ 技术的内容}

虚拟现实技术即 VR 技术, 是随着网络信息化时代的兴 起而产生的一种技术。起初人们只是设想能不能通过虚拟的 模拟, 将电脑上二维层面的图进行三维层面的展示, 后来这 种想法又被应用到影视动漫的制作中, 这种技术使那些平面 的人物拥有立体的身体, 随着时代的发展, 3D 动画也逐渐出 现在人们的眼球中。与此同时, 3D 时代也到来了, 越来越多 的 3D 技术和 $3 \mathrm{D}$ 软件开始出现, 最出名的就是近几年出现的 3D 打印技术了。总而言之, 抛弃以往那些旧的教学系统, 使 用新的网络信息化技术去教育建筑行业的学生们, 不但可以 把传统建筑教育在实体教学用具上的投资资金完全控制在 合理的范围之内, 减少了许许多多不必要的花费。还可以为 后期的 VR 技术的使用提供方便可行的方案, 更好地安排人 力、物力, 减少财政资金不必要的花费, 而且更好地扩大化教 育事业单位资产的投资效益。因此, 只有全面、细致、规范 的去规划好虚拟技术在建筑工程上的应用, 才能加强事业单 位对教学进度的控制, 使教育事业单位的固定资产在一开始 就走上规范、合理的路线, 也为业内稂莠不齐的教育改革方 案提供新思路。

\section{$2 \mathrm{VR}$ 技术应用在建筑设计教学中的结构}

为了初步探索 VR 技术在建筑工程教学中的应用, 我们 首先开展一门选修课, 作为 VR 技术应用的实验基地。只有通
过试点的初步总结, 我们才有把握能够进行大方面的课程推 广。为了使 VR 技术也能体现出建筑设计的系统教学内容, 我们首先在知识层面上, 将建筑设计课程体系形成两大类的 教学板块。根据一般的设计培养方案, 在实验教学等方面, 将原来的课程和实践理论基础分为 “实验理论学习” 和 “知 识体系实践学习” 两大板块。其次, 我们再将 VR 技术模拟建 筑设计课分为三等, 依次为基础型设计课程、综合型设计课 程和设计创新型实践。由此这般可以先让学生们进行参考, 然后进行学习, 且每一等设计课程都设置严格的考核制度, 一旦不通过则无法进入下一等的学习 ${ }^{[1]}$ 。因材施教, 才能走 得更长远。其中, 基础型设计课程主要是培养学生的基础知 识, 科目不难但是需要人博闻强记, 这是为学生们打好基础 的第一步, 也是考验学生学习能力的时候。综合型设计课程 则比基础型的要复杂一些, 需要学生进行全面的考虑, 包括 学生设计想法和设计合理性的考验,一般来说这一步的学生 都具有较好的学习能力。而设计创新实验, 则需要考察学生 的灵活性, 除了扎实的理论知识还有过人的创新性, 这个要 求一般比较高。

\section{3 从 VR 技术的层面上,构建了相对独立的实验教学教材}

VR 技术说难也不难, 但是说简单也是绝对不简单的。它 的受众群体主要是一些年轻的学生或者老师, 而且老师的接 受能力可能还比学生差很多, 因此在 VR 技术教学时, 我们除 了要交给学生的基础学习知识, 我们还要提前对老师进行 VR 技术的培训。对于那些年老的、难以接受新鲜事物的老师来 说, 学习VR技术是难度较大的, 因此我们可以暂时不让 VR 技 术掺入它的课程, 但是我们还是培训年轻老师对该课程的 VR 技术掌握。从老师到学生的自上而下的学习方式, 才能真正 的保证 VR 技术能够被教授给学生, 且 VR 技术才能在建筑设 计的教学过程中起到真正的作用 ${ }^{[2]}$ 。当然, 最后的学习方式 是为该 VR 技术的使用, 设立出一个专门的课程, 以及为它做 出相对应的、独立的实验教材, 把 VR 技术的学习也当成一门 必修课。开始可能会很艰难, 但是时间长了, 我们就会发现 VR 技术能为我们的建筑设计学习与工作提供极大的方便, 不 
可能忽略它的作用。

\section{4 构建了多样化的教学方法与手段}

虽然融入了 VR 技术的教学方式, 为一些不便进行空间 想象的建筑设计课程提供了方便, 但对于一些不够自律的学 习者来说并没有多大作用, 反而 VR 技术可能会为学生们这 些不负责任的态度惹上一些不必要的麻烦。事实也是如此, 很多家长都无法同意在孩子的学习过程中加入网络课堂, 这 显然是对推广VR 技术的一种障碍。因此, 我们需要设立一些 自我保护以及保护学生利益的手段, 尽力培养符合实践需 求、实践能力强、适应于这种教育模式的人。以实践教学改 革为切入点, 按照 “模拟、仿真” 的思路, 构建以模拟手段不 过分依赖网络的技能教学方法。建立起模拟科技的实验教学 环境, 采用模拟实验教学的模式, 使学生在一个严谨的环境 下学习。大大提高了学生和老师掌握 VR 技术和课程的学习 效果, 为学生进入后期的实践打下坚实的基础。

同时, 制定了多重的评分和考核标准, 该标准可以尽快 的与社会上专业建筑设计师的考试完全接轨。用于考核建筑 设计的实践技能教学效果, 达到以考促教, 以考促学, 充分发 挥技能考核的杜杆作用, 促进家长们对于该 VR 技术教学专 业性的信心。

\section{$5 \mathrm{VR}$ 技术在建筑设计教与学中的应用构建}

5.1 VR 技术、技能在建筑设计课程教学中的考核

很多人说, 基础知识牢固了整个学习过程就完成了百分 之九十, 但笔者觉得并非如此。建筑设计着重考察的是学生 的空间想象能力, 会死读书的人不一定比一些理论知识不够 牢固的人动手能力强, 反而在实际的建筑设计操作过程中表 现不好 ${ }^{[3]}$ 。因此, 加入 VR 技术的模拟教学考核也是非常重要 的一环, 我们可以通过模拟出来的建筑图纸来考察学生的动 手能力, 并通过询问的方式来考核学生的理论知识是否牢 靠。我们需要逐步考核, 分为平时考核和期末考核, 从而全面 的把握一个学生的能力。

\subsection{VR 技术毕业考试}

因为结合了 VR 技术的教学方式与传统的学习方式大不 相同, 我们也需要对使用 VR 技术进行教学的课程设置毕业 考试, 并对学生的实操能力进行部分的挑战。传统的教学方 式过于注重对学生讲述能力和理论知识的培训与考察, 但实 际的建筑设计工作岗位并不需要过多的理论知识。去建筑设 计院上岗, 他们更需要考核实际的操作能力。录用一个空有 证书却没有实际操作能力的人, 那将是非常可怕的, 那是对
公司的不负责, 也是对整个建筑的不负责。因此, 我们可以初 步考虑削减毕业考试中对学生理论知识的考核, 用更为实际 的操作手法考核和临时发挥来考验学生的专业素养。设置多 重考核, 完善评判标准, 同时安排多人进行考验, 与职业的建 筑设计师考核制度进行接轨。

\section{VR 技术实验教学体系构建的对外辐射作用}

将 VR 技术在建筑设计的教与学中进行应用, 无疑会在 长期以来十分平静的建筑设计教学体系里激起一番涟渏。在 初步得到上级领导和教育部的肯定之后, 我们可以进一步将 这一教学制度与其他的学校进行交流, 从中寻找优化方案并 对周围的教育体系产生辐射效应。一传十, 十传百, 最终能够 从根本上改变传统教学系统的弊病。首先, 我们要注意的就 是在进行全面的 VR 技术应用之前, 要考虑优选方案, 要求每 个参与人员都能提出自己的设计方案出来。因为有许多的参 与人员并不管投资方案是否对教育单位有很关键的作用, 他 们只管进行 VR 技术应用, 而不会做出其他合理的思考, 他们 缺乏经验却并不会主动去弥补这些缺陷, 因此这就使设计方 案和实际应用方面发生了脱节, 很难解决根本上的问题。因 此我们必须通过提高管理手段, 来增强教育单位的责任心, 促使这些管理人员主动去提高 VR 技术应用的质量, 可以用 奖励也可以用处罚制度。只有将他们自身的利益与整个单位 进行挂钩, 他们才会意识到自己应该做什么。

\section{7 结束语}

综合以上将 VR 技术应用到建筑设计教学过程中的改革 创新思路, 我们可以得知, 网络和现代化虚拟技术的应用对 建筑行业中的建筑设计实验教育的改革具有很高的实用性。 适当的处理就能够达到很好的教学效果, 还更能提高学生学 习的兴趣, 鼓励学生们自己去竞争, 自己去学会思考和学习, 而不是传统被动的接受。如果这一思路能够很好地对学生们 的学习起作用, 我们还可以将这一套方案进行改编, 并应用 到其他的建筑学专业课程里面, 这样更有意义。

\section{[参考文献]}

[1]华孟楠.虚拟现实技术在室内设计中的应用[J]. 北京 工业职业技术学院学报,2018(9):34.

[2]杨秀云.谈 VR 技术及其在高等教育领域的应用 [J].长 春师范大学学报(自然科学版),2017(4):148.

[3]朱殿骅.建筑教育制度改革目标与路径选择——基于 《虚拟技术应用》的视角[J].师范大学学报,2016(10):14-16. 